\title{
Unilateral Vogt-Koyanagi-Harada Disease: A Clinical Case Report
}

\author{
Arminda Neves $^{\mathrm{a}} \quad$ Ana Cardoso $^{\mathrm{b}}$ Mariana Almeida $^{\mathrm{a}} \quad$ Joana Campos ${ }^{\mathrm{a}}$ \\ António Campos ${ }^{a}$ João Paulo CastroSousa ${ }^{a}$ \\ ${ }^{a}$ Ophthalmology Department, Leiria Hospital Center, Leiria, and ${ }^{\mathrm{b}}$ Ophthalmology \\ Department, Local Health Unit of Baixo Alentejo, Beja, Portugal
}

\section{Key Words}

Unilateral Vogt-Koyanagi-Harada disease · Fundus fluorescein angiography · Corticosteroid therapy · Cyclosporine

\begin{abstract}
Purpose: To report a case of a 20-year-old female with decreased visual acuity (VA) in the left eye (LE). Methods: This is a retrospective and descriptive case report based on data from clinical records, patient observation and analysis of diagnostic tests. Results: A 20-year-old female presented with decreased VA in the LE for 3 days. Best-corrected visual acuity (BCVA) was 20/20 in the right eye (RE) and 20/40 in the LE. Pupillary function, intraocular pressure, results of external segment examinations and slit-lamp biomicroscopy were normal, bilaterally. RE fundoscopy was normal, and in the LE it revealed papillitis and posterior pole exudative retinal detachment. Optical coherence tomography (OCT) confirmed the macular serous retinal detachment and showed thickening of the posterior choroid also revealed by orbital ultrasound and magnetic resonance imaging (MRI). Fluorescein angiography showed angiographic features typical of Vogt-Koyanagi-Harada (VKH) disease: disseminated spotted choroidal hyperfluorescence and choroidal multifocal hypofluorescence, multifocal profuse leakage in the retina with pooling, serous retinal detachment and optic disc hyperfluorescence. Serological testing for the diagnosis of infectious pathologies was negative, and the review of systems was normal. The patient received systemic steroids and cyclosporine. LE BCVA improved up to $20 / 20$ at 18 months after the diagnosis, with complete reabsorption of subretinal fluid and normal retinal and choroidal thickness by OCT. Conclusion: Despite the unilateral involvement, the clinical and angiographic features were typical of VKH disease, and ophthalmologists should be aware to recognize this rare clinical variant of the disease.
\end{abstract}


Neves et al.: Unilateral Vogt-Koyanagi-Harada Disease: A Clinical Case Report

\section{Introduction}

Vogt-Koyanagi-Harada (VKH) disease is a multisystemic inflammatory autoimmune disorder that is characterized by panuveitis with iridocyclitis, serous retinal detachments, diffuse choroidal swelling and optic disc hyperemia. It may be associated with neurologic and cutaneous manifestations. There are 4 stages of VKH disease, including prodromal, acute uveitis, convalescent and chronic/recurrent uveitis; the clinical features of VKH vary depending on the disease stage $[1,2]$. The ocular manifestations are typically bilateral, but an asymmetric severity is usually present. There are few reports on unilateral VKH disease [35], and some of them are delayed forms of bilateral disease [6, 7]. Here, we describe a unilateral case of VKH disease.

\section{Case Presentation}

A previously healthy 20-year-old female presented to our emergency room with decreased visual acuity (VA) in the left eye (LE) for 3 days associated with a 3-week frontal headache. There was no history of penetrating ocular trauma or surgery. At the initial visit, the best-corrected visual acuity (BCVA) in the LE was 20/40 and in the right eye (RE) it was 20/20. Pupillary function, intraocular pressure and biomicroscopic examination were normal. RE fundoscopy was normal but fundus examination of the LE showed papillitis and posterior pole serous retinal detachment (fig. 1, left).

Optical coherence tomography (OCT) confirmed serous retinal detachment at the macular area with a retinal thickness of $388 \mu \mathrm{m}$ and thickening of the posterior choroid in the LE (fig. 1, right). A fundus fluorescein angiography (FA) revealed angiographic features typical of VKH disease in the LE: multifocal blocking of background fluorescence from the choroid, serous detachments with late pooling of dye, optic disc hyperfluorescence, focal areas of delayed choroidal perfusion and multifocal areas of pinpoint leakage with late pooling and leakage of dye in the subretinal space (fig. 2, left).

Markers of inflammation and autoimmunity were negative as were the serological testing for the diagnosis of infectious pathologies. The review of systems was normal.

Brain magnetic resonance imaging (MRI) showed an intraocular lesion in the LE corresponding to a focal thickening of the uveal tract of 3-4 mm (fig. 2, right). High-steroid pulse therapy (intravenous methylprednisolone $1,000 \mathrm{mg}$ for 3 days) was given. One week after treatment, an increase of the LE BCVA to 20/30 and a decrease in retinal $(281 \mu \mathrm{m})$ and choroidal thickness has been observed (fig. 3, top left).

The patient was prescribed with oral prednisolone $64 \mathrm{mg}$ once daily after breakfast on a tapering schedule with omeprazole $20 \mathrm{mg}$ for 10 weeks. Three weeks after the beginning of oral steroid therapy, the patient was prescribed with immunosuppressive medication during 11 months: cyclosporine $125 \mathrm{mg}$ twice daily for 4 months, and then she received a tapering schedule until $25 \mathrm{mg}$ once daily. Two months after the initial presentation, the LE BCVA increased to 20/20 and retinal thickness was $191 \mu \mathrm{m}$, with no thickening of the posterior choroid.

After 12 months of ongoing treatment (18 months after the initial clinical presentation), the LE BCVA kept on 20/20 with complete reabsorption of subretinal fluid, normal retinal and choroidal thicknesses confirmed by OCT (fig. 3, bottom left) and development of 'sunset glow' fundus in the affected eye (fig. 3, right), characteristic of VKH. Fundus appearance of the RE was normal (fig. 3, middle). 
Neves et al.: Unilateral Vogt-Koyanagi-Harada Disease: A Clinical Case Report

\section{Discussion}

Current diagnostic criteria for VKH disease require the presence of bilateral ocular involvement; however, unilateral or delayed involvement of the fellow eye can occur in rare cases [8]. The diagnosis of VKH disease is essentially clinical and angiographic: exudative retinal detachment during the acute disease is very specific and FA typically reveals numerous punctate hyperfluorescent areas at the level of the retinal pigment epithelium in the early stage of the exam followed by pooling of dye in the subretinal space. Differential diagnosis includes other causes of posterior/panuveitis, such as sympathetic ophthalmia, uveal effusion syndrome, posterior scleritis, primary intraocular lymphoma, uveal lymphoid infiltration, acute posterior multifocal placoid pigment epitheliopathy and sarcoidosis. VKH disease may be differentiated from other causes by a complete history, review of systems and physical examination associated with a laboratory evaluation [2]. In this case, all laboratory tests were negative and review of systems was normal, excluding other possible diagnoses. Despite the unilateral involvement, the clinical and angiographic features were typical of VKH disease. The patient did not develop dermatologic changes, which is consistent with previous data that most patients with VKH disease who are diagnosed and treated promptly never develop them [8]. The complaining of a 3-week history of frontal headache before the ocular manifestations is in accordance with the typical prodromal stage of the disease that includes flulike symptoms such as headache, nausea or fever [2].

Systemic steroids are the therapy of this entity in order to control active inflammation and to prevent new inflammatory episodes. They are tapered slowly to prevent recurrence of the disease and to minimize the incidence and severity of extraocular manifestations $[2$, 8]. However, there are recent studies which support the evidence that first-line use of corticosteroid combined with immunosuppressive agents decreases the development of late complications and recurrence of the disease, improves long-term vision and facilitates more rapid tapering of steroids [8-10]. Cyclosporine seems to be a better immunosuppressive agent than azathioprine, with good efficacy and safety [10]. Our patient received systemic corticosteroid therapy for 10 weeks and cyclosporine for 11 months. Eighteen months after the initial clinical presentation she was asymptomatic.

In VKH disease, the 'sunset glow' fundus (depigmentary changes in melanocytes in the choroid) is an important finding that appears between 2 and 6 months after disease onset [11]. The development of LE depigmentary changes in our patient, in comparison to normal fundus pigmentation in the RE, is one more clinical sign that supports the diagnosis of unilateral VKH.

There are few reports on unilateral VKH in the literature (less than 10), and some of them are delayed forms of bilateral disease [3-7]; our report adds one more case to this rare condition, with no clinical signs of the disease in the contralateral eye. Some of the published case reports had a final BCVA less than 20/20 in the affected eye; our case highlights the importance of a correct diagnosis and treatment to achieve a good recovery in VA.

In conclusion, our study presents a rare clinical variant of the VKH disease with completely recovery after treatment and enhances the fact that unilateral VKH disease is rare but should be promptly diagnosed and treated.

\section{Statement of Ethics}

The authors state that the subject of this article gave informed consent and the study protocol has been approved by the institute's committee on human research. 
Neves et al.: Unilateral Vogt-Koyanagi-Harada Disease: A Clinical Case Report

\section{Disclosure Statement}

The authors have no proprietary interests, financial support or other conflicts of interest to report.

\section{References}

1 Sakata VM, da Silva FT, Hirata CE, et al: Diagnosis and classification of Vogt-Koyanagi-Harada disease. Autoimmun Rev 2014;13:550-555.

2 American Academy of Ophthalmology - The Eye MD Association (2014): Vogt-Koyanagi-Harada syndrome; in Basic and Clinical Science Course, Section 9: Intraocular Inflammation and Uveitis, chapter 6: Noninfectious (Autoimmune) Ocular Inflammatory Disease. San Francisco, AAO 2014. pp 183-190.

-3 Forster DJ, Green RL, Rao NA: Unilateral manifestation of Vogt-Koyanagi-Harada syndrome in a 7-year old child. Am J Ophthalmol 1991;111:380-382.

-4 Usui Y, Goto H, Sakai J, Takeuchi M, Usui M, Rao NA, et al: Presumed Vogt Koyanagi Harada disease with unilateral ocular involvement: report of three cases. Graefes Arch Clin Exp Opthalmol 2009;247:1127-1132.

5 Agrawal A, Biswas J: Unilateral Vogt-Koyanagi-Harada disease: teport of two cases. Middle East Afr J Ophthalmol 2011;18:82-84.

-6 Kouda N, Sasaki H, Harada S, Yamada Y, Takahashi N, Sasaki K, et al: Early manifestation of Vogt Koyanagi Harada disease as unilateral posterior scleritis. Jpn J Opthalmol 2002;46:590-593.

7 Roe RH, Rathinam SR, Wong RW, McDonald HR, Jumper JM, Cunningham ET Jr: Delayed fellow eye involvement in patients with Vogt-Koyanagi-Harada disease. Br J Ophthalmol 2009;93:701-702.

-8 Cunningham ET Jr, Rathinam SR, Tugal-Tutkun I, Muccioli C, Zierhut M: Vogt-Koyanagi-Harada disease. Ocul Immunol Inflamm 2014;22:249-252.

-9 Paredes I, Ahmed M, Foster CS: Immunomodulatory therapy for Vogt-Koyanagi-Harada patients as first-line therapy. Ocul Immunol Inflamm 2006;14:87-90.

10 Cuchacovich M, Solanes F, Díaz G, et al: Comparison of the clinical efficacy of two different immunosuppressive regimens in patients with chronic Vogt-Koyanagi-Harada disease. Ocul Immunol Inflamm 2010;18:200-207.

11 Suzuki S: Quantitative evaluation of 'sunset glow' fundus in Vogt-Koyanagi-Harada disease. Jpn J Ophthalmol 1999;43:327-333.
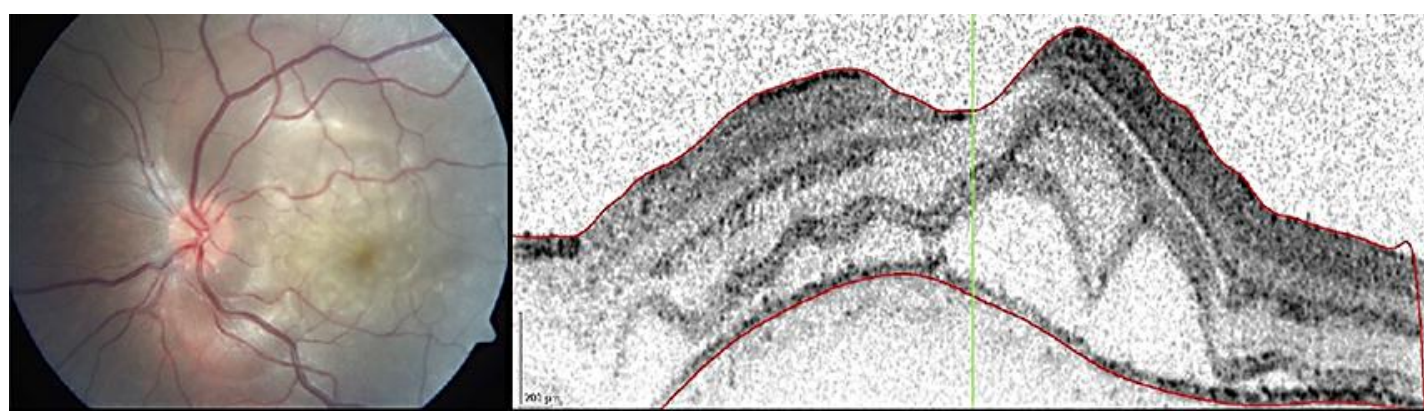

Fig. 1. Retinography and OCT of the LE at presentation, revealing papillitis and posterior pole exudative retinal detachment. Retinal thickness was $388 \mu \mathrm{m}$, and there was thickening of the posterior choroid. 


\section{Case Reports in \\ Ophthalmology}

\begin{tabular}{l|l}
\hline Case Rep Ophthalmol 2015;6:361-365 \\
\hline DOI: 10.1159/000441616 & $\begin{array}{l}\text { @ 2015 The Author(s). Published by S. Karger AG, Basel } \\
\text { www.karger.com/cop }\end{array}$ \\
\hline
\end{tabular}

Neves et al.: Unilateral Vogt-Koyanagi-Harada Disease: A Clinical Case Report

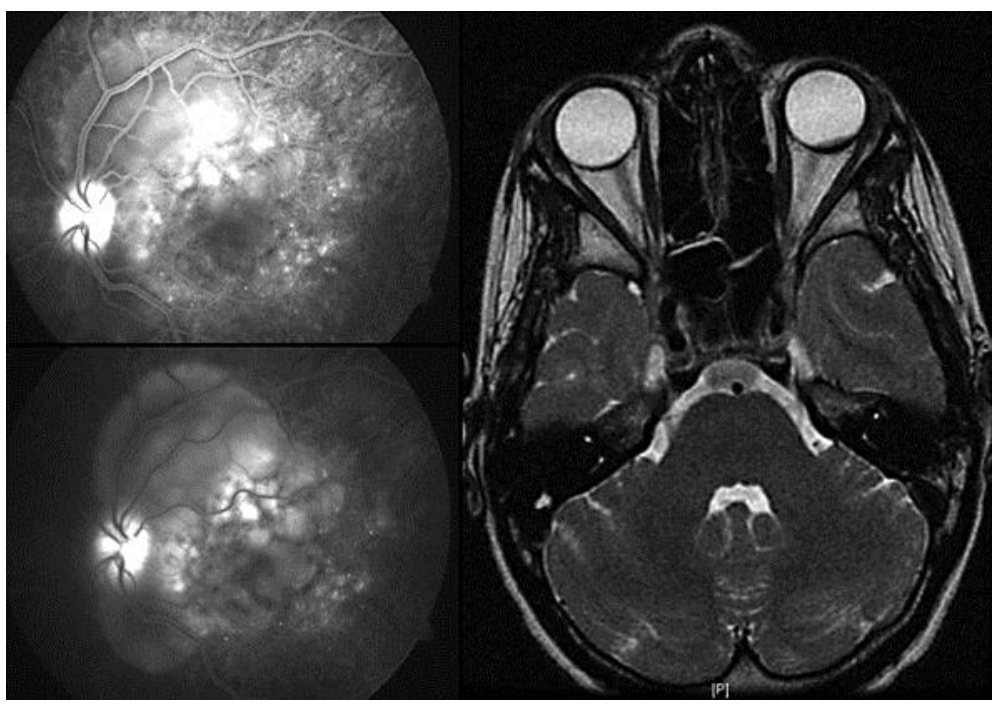

Fig. 2. FA of the LE at presentation (left) revealing focal areas of delayed choroidal perfusion and multifocal areas of pinpoint leakage in the early arteriovenous phase (top left), with pooling and leakage of dye in the subretinal space in the late stage of the angiogram (bottom left). Brain MRI showing a focal thickening of the uveal tract of 3-4 $\mathrm{mm}$ in the LE (right).

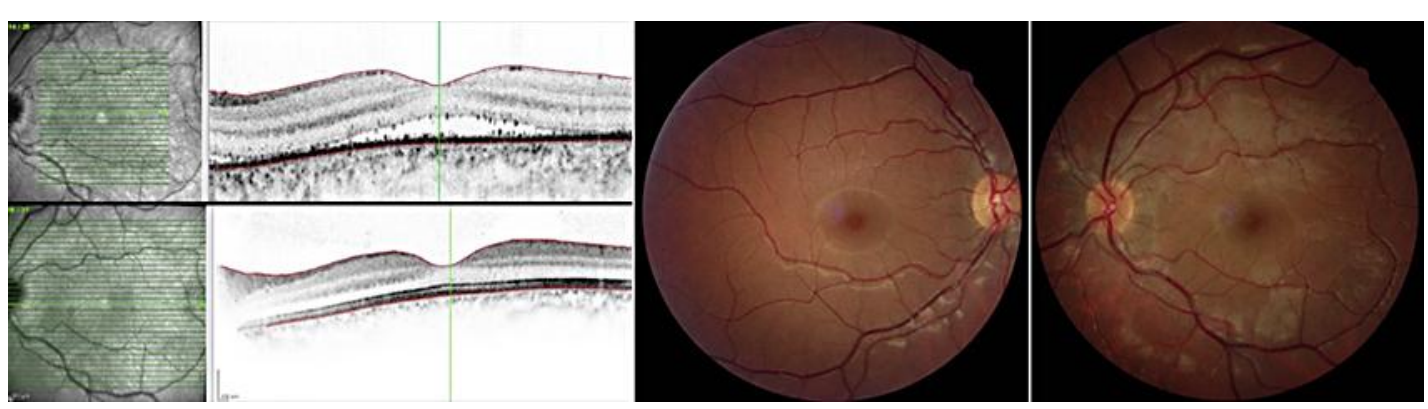

Fig. 3. OCT of the LE 1 week after treatment (top left) and 18 months after initial clinical presentation (bottom left), showing a decreased retinal and posterior choroidal thickness, with a 'sunset glow' fundus appearance at 18 months (right) in comparison to a normal fundus appearance in the RE (middle). 